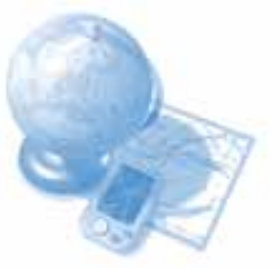

James Davis

Chemistry

School of Biomedical and

Natural Sciences

Nottingham Trent

University

Nottingham

NG11 8NS

james.davis@ntu.ac.uk

The basic rationale was to provide an integrated

mentor scheme that offers

a framework to

complement the

traditional Lecture-Lab-

Tutorial teaching

protocols that are

common to most science

degree programmes

\section{Assistive Leaming and Research Mentoring Schemes}

\begin{abstract}
The adoption of undergraduates into research teams upon entering university represents a marked change from the prescriptive lecture-lab format that underpins current teaching formats within the physical sciences. One such approach has been piloted at Nottingham Trent University - though not as a replacement for traditional teaching methods but rather to compliment and enhance the university experience for new entrant undergraduates. The programme has aimed to foster a student centred approach to their studies within chemistry through providing a genuine, real world context wherein they can tackle real problems that will help to reinforce the academic content and develop transferable skills. While the programme can be viewed as an enhanced work experience programme for undergraduates, its principal aim is to provide a pro-active mentoring framework that will nurture student enthusiasm for the subject. The logistics of running such a programme are outlined and the preliminary outcomes from the initial pilot are discussed.
\end{abstract}

\section{Introduction}

At present, most undergraduate research within university curricula is largely restricted to final year projects. There are good reasons for doing so, under the assumption that students will have gained, in the previous two years, the core knowledge that should enable them to function relatively independently within the laboratory environment. The project is widely perceived by students as being the most interesting part of the course largely as a result of the independence, the challenge of the new and the fact that the results obtained could have real world significance. Could the same not be applied to first and second year students? Assistive Learning and Research Mentoring is a Development Project funded by the Higher Education Academy that has sought to assess the potential impact of attempting to introduce entry level students to research as a means of enhancing their undergraduate experience.

\begin{tabular}{|c|c|c|}
\hline & $\begin{array}{c}\text { End of Yea } \\
\text { Grade }\end{array}$ & No. \\
\hline Chemistry & /st - & 3 \\
\hline \multirow{3}{*}{$\begin{array}{l}\text { Level 1: } 10 \\
\text { Level 2: } \quad 4\end{array}$} & 2.2 & 1 \\
\hline & 3rd - & 3 \\
\hline & $\begin{array}{ll}1 \text { st } & - \\
2.1 & -\end{array}$ & $\begin{array}{l}1 \\
3\end{array}$ \\
\hline \multicolumn{3}{|l|}{ Forensic Science } \\
\hline \multirow[t]{2}{*}{ Level 2: 6} & $2.1-$ & 3 \\
\hline & Total & 20 \\
\hline $\begin{array}{l}\text { Figure 1: Academic ra } \\
\text { the first year for stud }\end{array}$ & $\begin{array}{l}\text { sing at the } \\
\text { ts in pilot }\end{array}$ & $\begin{array}{l}\text { end of } \\
\text { tudy }\end{array}$ \\
\hline
\end{tabular}

The basic rationale was to provide an integrated mentor scheme that offers a framework to complement the traditional Lecture-Lab-Tutorial teaching protocols that are common to most science degree programmes. It was hoped that the scheme would provide students with a mechanism through which they could, to a limited extent, direct their own studies within an easily identifiable real world context. The approach taken here has been to adopt undergraduates into active research groups and encourage their direct participation in a range of multi-disciplinary projects. This offers an opportunity to provide 
excitement in a way that is immediate, upon entry to the course, tangible, through the development of key skills, teamworking and real world problem solving, and desirable, by providing individual identities and visible career enhancement. The distinction between school and university is clarified and, with the opportunity to participate in something real, a sense of importance can be fostered that should enhance the esteem of the individual student. Buddy schemes have been used extensively as a means of bridging the school/university transition for new entrants, but these often provide little more than an opportunity for social familiarisation with the campus setting. It was anticipated that the research mentoring programme, in contrast to undergraduate pairing, would provide a more structured support network that could serve to counter or alleviate the more academic concerns of those new to the university.

There are obvious problems in the implementation of such programmes: the limited scientific background of the student, the availability of instrumentation, health and safety considerations, the possible expense and the increased time demands placed on the supervisor. When would it be done and would it require additional timetabling? How would it impact upon the student workload? These are the main questions that the project has sought to address. The project has an initial lifetime of three years, such that the progress of students from Year 1 through to graduation could be followed. At present, it has been running for almost two of the three years originally specified and a preliminary evaluation of the scheme and its participants is now presented.

\section{Project Methodology}

The basic plan was to allow students an opportunity to engage in a number of distinct research projects. Recruitment to the scheme was purely voluntary, was not assessed and no additional timetabling was required. Irrespective of course programme, there will inevitably be periods where the students have blocks of free time. In our experience this is usually the Wednesday afternoon traditionally reserved for those with an interest in sports. Students were assigned a project and, after an initial induction period, were given a particular part of the project on which to work, through which they would engage in the process of research. Supervision was based on the close interaction between student and postdoctoral or postgraduate supervisor, who would then report to the academic supervisor. The overall aim is to foster an effective working partnership in which the student actively contributes to the group.
The scheme essentially mirrors a work experience programme but one where the outcome and direction can be dictated by the student, albeit with a modicum of surreptitious academic guidance. The students are provided with an arena that can enable them to place their studies in context and to hone their skills on something more tangible than that offered by library study and recipe based practical sessions. The programme is run as a complementary activity to the existing laboratory work and is not intended to replace the latter. The key advantage is

that as there is no formal assessment, the pressure of failing is removed and the burden of 'mistakes' is shared by the supervising researchers in an environment that actively promotes a supportive teamworking and problem solving ethos that enhances the development of key skills. It would be hoped that the improved confidence generated by the additional laboratory work would provide a positive feedback into the programmed laboratory sessions.

\section{Project Implementation}

A total of 20 students have engaged in the pilot project since its inception in October 2005.

These were drawn

predominantly from the

Chemistry cohort with six students recruited from the Forensic Science programme. This accounts for approximately $8 \%$ of the new entrants within the chemistry stream. The breakdown of the respective groups and their academic ranking at the end of the first year is detailed in Figure 1. It is clearly not possible to let the students simply run free within the laboratory environment. Adoption within a research group however could essentially overcome many of the negatives highlighted previously. A key tenet is that the research and support staff are willing to supervise the students during their time within the laboratory and hence maintain compliance with appropriate health and safety practices. The academic should thereby be released from the demands of having continually to watch over the students while the students gain the freedom to experiment and develop their skills without the consequences of formal assessment.

An assumption is that the supervisory duties can be distributed amiably amongst the research staff (postgraduate and postdoctoral). In principle, it could be an effective means of providing those players with an opportunity to develop their own mentoring skills. In so doing, it could be a highly effective vehicle for promoting 'Life Long Learning' staff development goals. In practice, this will obviously be dependent on the individual concerned and their own workload. In the majority of cases, the opportunity to supervise students has been met with a high degree of enthusiasm. It has to be acknowledged that in the initial phase there is a large investment in time on 
the part of the supervisory team to bring the students up to speed with the running of the particular laboratories, establishing competency in the various tasks and on the specific demands of the project itself. In the long run however, this can be largely offset as the new entrants become the supervisors in the second year. In principle there is the potential for a self perpetuating cycle that actively reinforces the student confidence for new entrants and established members.

The ability and desire of students to participate within a research environment is woefully undervalued. The enthusiasm and inevitable 'but why?' questioning can, with a little imagination and appreciation of the students' educational level, be harnessed to benefit most research processes irrespective of division. This is not to say that they can be immediately engaged in cutting edge science, but there is always a role for smaller, proof of concept, projects. The interaction of the various groups also brings the benefits of developing much heralded transferable skills (teamworking, problem solving, communication, computing) and can also foster a competitive edge that helps to drive the students and the project forwards. Many courses place great emphasis on their 'Scientific Communication' modules and this scheme can clearly form synergistic relationships across a range of modules with the research project providing a strong contextual basis.

\section{Project Evaluation}

The project is being evaluated on a number of levels and includes: determining the type of student that opts for the programme; the weekly attendance of the students and the impact of coursework on this, the attrition rate over the year; continuation into subsequent years; the possible influence that the additional training has on placement interviews and the impact on their academic performance (laboratory and final exam). The low sample number will create an obvious problem when attempting to ascribe statistical significance to any trends that emerge from those participating in the programme in comparison with the bulk of the chemistry cohort. To counter this, at least in part, the programme is being evaluated over an initial three year period which should give a more detailed assessment of how the students have progressed over the entire programme. It can be seen from Figure 1 that it appears to cater for the more gifted student despite the fact that it was open to all and there was no discrimination in the recruitment process. It is clear however that the programme self-selects and a sad outcome is the fact that it is largely ignored by those who could benefit the most in terms of the need to improve academic performance and laboratory skills. This induces an obvious problem in attempting to elucidate the influence on academic performance beyond the anecdotal as it could be argued that the students would have achieved similar results even if they not taken part in the programme.

\section{Preliminary Conclusions}

A number of reasons can be attributed to the bias in the recruitment statistics: the principal factor may however be the perception of the increased workload. It could be anticipated that the more able students feel confident in juggling the additional commitment. Part time employment must also be considered and will impact on student participation. The fact that the programme runs within the existing curricula can, in part, offset that potential conflict with part time employment.

Nevertheless, it is an important factor that imposes increasing time constraints on students and it must be acknowledged that the majority of those taking part were not subject to the demands of evening or weekend employment. The programme clearly provides a worthwhile contribution to the student experience, as evidenced by the relatively small attrition rate over the first year. The project has also realised tangible and indeed notable results. A number of research publications ${ }^{1,2}$ have arisen in the course of the first year work and students have won a number of prizes at conferences at national and regional level for both poster ${ }^{3}$ and oral $^{4}$ contributions. In response to the preliminary evaluation, the second phase of the programme has been to actively encourage the participation of weaker students, especially those entering their second year. This group may provide a stronger foundation for assessment of the potential impact on academic performance as their initial, first year, results will provide a more reliable benchmark. The fact that the first year of the programme has given rise to recognisable outcomes that can significantly enhance the student $\mathrm{CV}$ can be used to advertise the merits of participation in contrast to a view of the programme as simply yet more coursework.

\section{Acknowledgements}

The author thanks the Higher Education Academy Physical Sciences section for sponsoring the pilot study. 


\section{References}

1. Smith, R. B., Canton, C., Lawrence, N. S., Livingstone, C., Davis, J. (2006) Molecular anchors - mimicking metabolic processes in thiol analysis, New Journal Chemistry, 30, 1718-1724.

2. Marti, M., Litchfield, V., Smith, R. B., Franklin, A. M., Livingstone, C., Davis, J, A Chromatographic Tool for Preparing Combinatorial Quinone - Thiol Conjugate Libraries, Journal Biochemical and Biophysical Methods, in press.

3. 2nd National FORREST

Conference 2006.

(FORensic RESearch and Teaching). $12^{\text {th }}-14^{\text {th }}$ Sept 2006. University of Central Lancashire.

4. RHINE 2006, RSC Regional Electrochemistry Meeting, Dec 2006, University of Hull.

The programme

clearly provides a

worthwhile

contribution to the

student experience, as

evidenced by the

rela tively small attrition

rate over the first year. 